\title{
Hamiltonian Collapsing of Irrational Lagrangian Submanifolds with Small First Betti Number ${ }^{\star}$
}

\author{
François Lalonde \\ Département de Mathématiques et d'Informatique, Université de Québec à Montréal, \\ C.P. 8888, Succ. A, Montréal, H3C 3P8, Québec, Canada
}

Received August 26, 1991; in revised form March 23, 1992

\begin{abstract}
Among the main symplectic invariants of a closed Lagrange submanifold $L$ of the cotangent of $\mathbb{R}^{n}$ is the tubular radius $R(L)$ defined as the smallest tube $D(r) \times \mathbb{C}^{n-1}$ of $\mathbb{C}^{n} \simeq T^{*} \mathbb{R}^{n}$ in which $L$ can be pushed by an Hamiltonian diffeotopy of $\mathbb{C}^{n}$. We show here, using pseudo-holomorphic techniques, that such a submanifold cannot collapse if the first Betti number of $L$ is smaller than 3 and if the Maslov class of $L$ does not vanish; in other words, $R(L)$ is then strictly positive and one can actually give an explicit lower bound in terms of the Liouville and Maslov classes of $L$.
\end{abstract}

\section{Introduction}

The study of symplectic invariants of closed Lagrangian submanifolds of cotangent spaces - an essential aspect of the theory of Hamiltonian systems - was confronted by some fundamental difficulties that have been partly understood during the last years. Two different approaches have been used yielding surprisingly similar estimates. The variational approach, for instance, has been used by Floer, Hofer, and Viterbo to obtain explicit values of the "capacity" of some Lagrangian submanifolds and lower bounds on the value of the "tubular capacity" of any Lagrangian embedding of the torus $T^{n}$ in $\mathbb{C}^{n}$. Here the capacity $c(E)$ of a subset $E$ of $\mathbb{C}^{n}$ is defined by a minimax procedure (see $[2,3]$ ) and is given, in the simplest case of a closed convex hypersurface of $\mathbb{C}^{n}$ by the smallest symplectic area of a closed characteristic; the tubular capacity $R(E)$ is the smallest radius of a tube into which $E$ can be pushed by an Hamiltonian diffeotopy:

$$
\begin{gathered}
R(E)=\inf \left\{r \in \mathbb{R}^{+} \text {such that there exists an Hamiltonian diffeomorphism } \phi \text { of } \mathbb{C}^{n}\right. \\
\text { with } \left.\phi(E) \subset D(r) \times \mathbb{C}^{n-1}\right\},
\end{gathered}
$$

where $D(r)$ is the open disk of radius $r$ of $\mathbb{C}$.

\footnotetext{
^ Partially supported by Research Grants NSERC OGP0092913 and FCAR EQ3518
} 
On the other hand, the elliptic approach, which relies on Gromov's theory of pseudoholomorphic curves, was the main ingredient in the recent proof by Sikorav [11] which gave explicitly a lower bound for the tubular capacity of any "rational" Lagrangian submanifold of $\mathbb{C}^{n}$ (see below); in the same spirit, the "disjunction energy" of rational Lagrangian submanifolds has been studied in a recent work by Polterovich ([10]). For a subset $E$ of $\mathbb{C}^{n}$, this is the minimal energy $e(E)$ needed to move $E$ to a position disjoint from its initial position (see $[7,12]$ ).

Let $L \subset \mathbb{C}^{n}$ be a closed Lagrangian submanifold. Since any symplectic diffeomorphism of $\mathbb{C}^{n}$ is Hamiltonian, $c(L), e(L)$, and $R(L)$ are symplectic invariants of the Lagrangian embedding $L \hookrightarrow \mathbb{C}^{n}$. These three invariants are related by:

$$
c(L) \leq e(L) \leq \pi R^{2}(L)
$$

Despite the importance of these invariants in Hamiltonian systems and symplectic topology, not much is known about their values for a generic Lagrangian submanifold; for instance, we do not know, in general, whether they vanish or not. The sole results up to now are, as we mentioned above, those obtained by Floer, Hofer, and Viterbo using a variational approach, and by Sikorav using elliptic methods:

1) If $T^{n} \hookrightarrow \mathbb{C}^{n}$ is a standard torus $S^{1}\left(r_{1}\right) \times \ldots \times S^{1}\left(r_{n}\right)$, then

$$
c\left(T^{n}\right)=\min _{1 \leq i \leq n} c\left(S^{1}\left(r_{i}\right)\right)=\pi\left(\min r_{\imath}\right)^{2},
$$

(product formula [5])

so that $R\left(T^{n}\right) \geq \min r_{i}$ and therefore $R\left(T^{n}\right)=\min r_{i}$. Moreover, Viterbo ([14]) recently showed that $R\left(T^{n}\right)>0$ for any Lagrangian embedding of the torus into $\mathbb{C}^{n}$. The proof gives $R\left(T^{n}\right)$ as being approximately equal to the largest radius of a Weinstein tubular neighbourhood of $T^{n} \hookrightarrow \mathbb{C}^{n}$.

2) Since the Liouville form of $\mathbb{C}^{n}, \lambda=\sum_{i=1}^{n} y_{i} d x_{i}$, is a primitive of the standard symplectic form, its restriction to any Lagrangian submanifold $L$ defines a DeRham cohomology class of $L$, called the Liouville class $\lambda(L)$. Now, if $L \hookrightarrow \mathbb{C}^{n}$ is a rational Lagrangian submanifold, that is whose Liouville class $\lambda(L)$ equals the product of a real scalar by an integral form, then it has been shown by Sikorav that

$$
R(L) \geq \sqrt{\varrho / \pi}
$$

where $\varrho$ is the largest real number such that $\lambda(L)=\varrho \times$ (integral class) (see [11]).

The aim of this paper is to show how elliptic methods (pseudoholomorphic curves) can be used to obtain explicitly a positive lower bound for $R(L)$ for any (rational or not) Lagrangian embedding of a manifold whose first Betti number is smaller than 3 , whenever its Maslov class does not vanish (the Maslov class $\mu(L) \in H^{1}(L ; \mathbb{Z})$ of a Lagrangian submanifold $L$ of a cotangent space measures the rotation along any loop of $L$ of the tangent space to $L$ with respect to the fiber of the cotangent bundle; in the euclidian case, this is simply the pull-back by the Gauss map of the generator of $H^{1}(\Lambda ; \mathbb{Z})$, where $\Lambda$ is the grassmannian manifold of Lagrange subspaces of $\mathbb{C}^{n}$ : see [1]). In the rational case, this lower bound improves the one given in (1). This result is a corollary of the following:

Theorem 1. Let $L \hookrightarrow \mathbb{C}^{n}$ be a closed Lagrangian submanifold of $\mathbb{C}^{n}$. If the restriction $\left.\lambda(L)\right|_{\operatorname{ker} \mu(L)}$ of the Liouville form to the kernel of the Maslov class is rational, then 
$R(L)>0$ and one can compute explicitly a lower bound of $R(L)$ in terms of $\lambda(L)$ and $\mu(L)$.

Corollary. $R(L)>0$ for any Lagrangian embedding with non-vanishing Maslov class of a closed manifold whose first Betti number is smaller than 3. In particular, this is true of any Lagrangian embedding of a manifold admitting a metric with non-positive curvature, with $\beta_{1}<3$.

If $\beta_{1}(L) \leq 2$ and $\mu(L) \neq 0$, the kernel of $\mu(L)$ has rank not greater than 1 , thus the form $\left.\lambda(\bar{L})\right|_{\operatorname{ker} \mu(L)}$ is rational.

Examples. Among the closed manifolds admitting a Lagrangian embedding in $\mathbb{C}^{n}$ and satisfying the hypotheses of the Corollary, there are $T^{2}$ and all manifolds of the form $S^{1} \times K_{1}$ and $T^{2} \times K_{2}$, where $K_{i}(i=1,2)$ is a manifold with $\beta_{1}\left(K_{\imath}\right) \leq 2-i$ admitting a metric with non-positive curvature and whose tangent bundle has a trivial complexification. In particular, every hyperbolic 3-manifold with $\beta_{1} \leq 1$ gives rise to such manifold after product with $S^{1}$ or $T^{2}$.

\section{Proof of the Theorem}

The proof relies on Gromov's theory of pseudoholomorphic curves: to begin with, we will put together the area estimates computed by Sikorav [11] and the bounds on Maslov indexes obtained by Polterovich [9] when a compactness phenomenon gives rise to families of pseudoholomorphic disks whose boundaries lie on a Lagrangian submanifold. In fact, here, these estimates will be worked out for a generic almost complex structure on $\mathbb{C}^{n}$ (sufficiently close to the standard one).

Let $L_{r}$ be a closed Lagrangian submanifold of $\mathbb{C}^{n}$ included in $D(r) \times \mathbb{C}^{n-1}$. We follow first Gromov's [6] and Polterovich's [9] argument. After a slight perturbation of $L_{r}$, we may assume that there exists a neighbourhood $\mathscr{\mathscr { C }}$ of $L_{r}$ and a $J_{0}$-antiholomorphic involution $\tau: \mathscr{\mathscr { C }} \rightarrow \mathscr{\mathscr { C }}$ whose fixed point set is $L_{r}\left(J_{0}\right.$ is the standard complex structure on $\mathbb{C}^{n}$ ). Denote by $\mathscr{J}$ the space of $C^{\infty}$ almost complex structures $J$ on $\mathbb{C}^{n}$ uniformly compatible with the standard symplectic structure $\omega$ of $\mathbb{C}^{n}$ [that is: $\omega(J v, J w)=\omega(v, w)$ and $\|v\|_{J}^{2}=: \omega(v, J v) \geq C_{J}\|v\|^{2}$ for all $v, w \in T_{*} \mathbb{C}^{n}$, where $C_{J}$ is a positive constant and \|\|$=:\|\|_{J_{0}}$, such that $\tau$ is $J$-anti-holomorphic, and whose Floer norm $\|J\|_{\varepsilon}$ is finite. See [4, Sect.5], for the definition and properties of the Floer norm: in fact, our space $\mathscr{J}$ is a large subspace of $C^{\infty}$ perturbations of $J_{0}$ defined as the image under the exponential map $T_{J_{0}} \overline{\mathscr{J}} \rightarrow \overline{\mathscr{J}}(\overline{\mathscr{J}}=$ all uniformly compatible $C^{\infty}$ almost complex structures such that $\tau$ is anti-holomorphic) of a small ball centered at the origin in the subspace $T_{J_{0}}^{\varepsilon} \overline{\mathscr{J}}$ of $T_{J_{0}} \overline{\mathscr{Z}}$ consisting of all $j \in T_{J_{0}} \overline{\mathscr{Z}}$ such that

$$
\|j\|_{\varepsilon}=\sum_{k \geq 0} \varepsilon_{k} \max _{z \in \mathbb{C}^{n}}\left|D^{k} j(z)\right|
$$

is finite, where $\varepsilon=\left(\varepsilon_{k}\right)_{k \in \mathbb{N}}$ is a sequence of positive real numbers. Lemma 5.1 of [4] shows that one can choose the sequence $\varepsilon$ in a such way that the Banach space $\left(T_{J_{0}}^{\varepsilon} \overline{\mathscr{J}},\|\|_{\varepsilon}\right)$ is sufficiently large.

For a fixed number $d \in(0,1)$ and any non-zero class $\alpha \in H_{1}\left(L_{r} ; \mathbb{Z}\right)$ define

$$
\begin{gathered}
\mathscr{F}_{\alpha}=\left\{f:\left(D^{2}, \partial D^{2}\right) \rightarrow\left(\mathbb{C}^{n}, L_{r}\right) \mid f \text { is of Hölder class } C^{2+d},\left[\left.f\right|_{\partial D^{2}}\right]=\alpha\right. \\
\text { and there exists } \left.z \in \partial D^{2} \text { such that } f^{-1}(f(z))=z \text { and } D f(z) \neq 0\right\} .
\end{gathered}
$$


Let $\mathscr{C}_{a}=\left\{(f, J) \in \mathscr{F}_{a} \times \mathscr{J} \mid \bar{\partial}_{J} f=0\right\}$ be the subspace of $\mathscr{F}_{\alpha} \times \mathscr{J}$ of all $J$ holomorphic disks whose boundaries lie in $\alpha$, where $\bar{\partial}_{J}=\frac{1}{2}\left(\frac{\partial}{\partial x}+J \frac{\partial}{\partial y}\right)$ is the Cauchy-Riemann operator for the structures $i=\sqrt{-1}$ on $D^{2}$ and $J$ on $\mathbb{C}^{n}$. Then $\mathscr{C l}_{a}$ is a Banach submanifold of $\mathscr{F}_{\alpha} \times \mathscr{J}$ (see [8] for instance) and the projection on the second factor $\pi_{\alpha}: \mathscr{M}_{\alpha} \rightarrow \mathscr{J}$ is a Fredholm operator of index $\mu(\alpha)+n$, where $\mu \in H^{1}\left(L_{r}: \mathbb{Z}\right)$ is the Maslov class of $L_{r}$. If $J$ is a regular value and $\pi_{\alpha}^{-1}(J)$ is not empty, then:

$$
3 \leq \operatorname{dim} \pi_{\alpha}^{-1}(J)=\operatorname{ind}\left(\pi_{\alpha}\right)=\mu(\alpha)+n,
$$

since the conformal group of the disk acts freely on $\pi_{\alpha}^{-1}(J)$, and thus $\mu(\alpha) \geq-n+3$. We will fix below a value $J$ close to $J_{0}$, and regular for all projection $\pi_{\alpha}$ corresponding to non-zero classes $\alpha$.

Finally, for each $J \in \mathscr{J}$ and each class $\beta \in H_{1}\left(L_{r} ; \mathbb{Z}\right)$, define

$$
\tilde{\mathscr{F}}_{\beta}=\left\{f:\left(D^{2}, \partial D^{2}\right) \rightarrow\left(\mathbb{C}^{n}, L_{r}\right) \mid f \in C^{2+d} \text { and }\left[\left.f\right|_{\partial D^{2}}\right]=\beta\right\}
$$

We wish to define properly the codomain of the elliptic operator $\bar{\partial}_{J}$ on $\tilde{\mathscr{F}}_{\beta}$. Since the quotient of the bundle of real homomorphisms $\operatorname{Hom}_{\mathbb{R}}\left(T \mathbb{C}, T \mathbb{C}^{n}\right) \rightarrow \mathbb{C} \times \mathbb{C}^{n}$ by the subbundle of the $(i, J)$-linear ones may of course be identified with the $(i, J)$-antiholomorphic ones and therefore to the trivial bundle $\left(\mathbb{C} \times \mathbb{C}^{n}\right) \times \mathbb{C}^{n} \rightarrow\left(\mathbb{C} \times \mathbb{C}^{n}\right)$, we define:

$\mathscr{G}=\left\{g: \mathbb{C} \times \mathbb{C}^{n} \rightarrow \mathbb{C}^{n} \mid g \in C^{\infty},\|g\|_{\varepsilon}<\infty\right.$ (Floer norm), and for $g$ viewed as a section of the bundle of $(i, J)$-anti-holomorphic homomorphisms, $g$ is $\left(\tau_{0}, \tau\right)$-invariant on $\mathscr{C b}_{0} \times \mathscr{W}$, where $\mathscr{C}_{0}$ is a neighbourhood of $\partial D^{2} \subset \mathbb{C}$ and $\tau_{0}: \mathscr{C}_{0} \rightarrow \mathscr{C}_{0}$ is an $i$-anti-holomorphic involution $\}$.

Let $\mathscr{N}_{\beta}$ be the subset of $\mathscr{\mathscr { F }}_{\beta} \times \mathscr{G}$ of all pairs $(f, g)$ such that $\bar{\partial}_{J} f$ is equal to the restriction of $g$ to the graph of $f$. Then the projection on the second factor

$$
\bar{\partial}_{J}: \mathscr{N}_{\beta} \rightarrow \mathscr{G}
$$

is a Fredholm operator of index $\mu(\beta)+n$. Suppose $\gamma$ is a path in $\mathscr{G}$ from 0 to a regular value $g$ such that $\bar{\partial}_{J}$ is transversal to $\gamma$. If $Y_{\beta, \gamma}=\bar{\partial}_{J}^{-1}(\gamma) \neq \emptyset$, then

$$
0 \leq \operatorname{dim} Y_{\beta, \gamma}=\operatorname{ind}\left(\bar{\partial}_{J}\right)+1=\mu(\beta)+n+1
$$

and therefore $\mu(\beta) \geq-n-1$ whenever $Y_{\beta, \gamma}$ is not empty.

Let us now follow Gromov's argument [6]: set $\beta=0$, there exist an open neighbourhood $U$ of $J_{0}$ in $\mathscr{J}$ and an open set $V$ of $\mathscr{G}$ such that the equation $\bar{\partial}_{J} f=g$ has no solution on $U \times \mathscr{N _ { 0 }} \times V$. We will specify this open set $V$. But first note that the equation $\bar{\partial}_{J_{0}} f=(c, 0, \ldots, 0)$ has no solution on $\mathscr{F}_{0}$ if $\|c\|$ is sufficiently large: this equation implies that $f$ is harmonic, and applying $\bar{\partial}_{J_{0}}$ to the Poisson formula restricted to the first component $f_{1}$ of $f$ and evaluating at $z=0$, we obtain:

$$
\bar{\partial}_{J_{0}} f_{1}(0)=\frac{-1}{2 \pi} \int_{0}^{2 \pi} e^{i \theta} f_{1}\left(e^{\imath \theta}\right) d \theta
$$


thus $\left|\bar{\partial}_{J_{0}} f_{1}(0)\right| \leq \max _{0 \leq \theta<2 \pi}\left|f_{1}\left(e^{\imath \theta}\right)\right|<r^{\prime}<r$, where $r^{\prime}=\frac{r+\bar{r}}{2}$ is the average of $r$ and the smallest radius $\bar{r}$ of a closed tube $\bar{D}(\bar{r}) \times \mathbb{C}^{n-1}$ containing $L_{r}$ [note that $r>\bar{r}$ because $L_{r}$ is compact and $D(r)$ is open]. There is therefore no solution to $\bar{\partial}_{J_{0}} f=(c, 0, \ldots, 0)$ when $\|c\| \geq r^{\prime}$. Without an a priori bound on the norm of $D f$, this might not remain true after a slight perturbation of the almost complex structure. This bound is given by the condition $f \in \widetilde{\mathscr{F}}_{0}$ :

$$
\begin{aligned}
\|D f(z)\|_{J}^{2} & =\frac{1}{4}\left(\left\|\frac{\partial f}{\partial x}(z)\right\|_{J}^{2}+\left\|\frac{\partial f}{\partial y}(z)\right\|_{J}^{2}\right) \\
& =\frac{1}{2}\left\langle J \frac{\partial f}{\partial x}(z), \frac{\partial f}{\partial y}(z)\right\rangle_{J}+\frac{1}{4}\left\|\frac{\partial f}{\partial x}(z)+J \frac{\partial f}{\partial x}(z)\right\|_{J}^{2} \\
& =\frac{1}{2}\left(f^{*} \omega\right)(z)\left(\frac{\partial}{\partial x}, \frac{\partial}{\partial y}\right)+\left\|\bar{\partial}_{J} f(z)\right\|_{J}^{2}
\end{aligned}
$$

which implies that $\int_{D^{2}}\|D f\|_{J}^{2}=\int_{D^{2}}\left\|\bar{\partial}_{J} f\right\|_{f}^{2}$ if $f \in \tilde{\mathscr{F}}_{0}$. Set

$$
\begin{aligned}
U=\left\{J \in \mathscr{V} \mid\left\|\bar{\partial}_{J} f-\bar{\partial}_{J_{0}} f\right\|<\varepsilon_{1}\|D f\| \text { for all } f,\right. \\
\left.\quad \text { and }\left(1+\varepsilon_{2}\right)^{-1}\|v\|<\|v\|_{J}<\left(1+\varepsilon_{2}\right)\|v\| \text { for all } v \in T_{*} \mathbb{C}^{n}\right\}
\end{aligned}
$$

and

$$
\begin{aligned}
& V=\left\{g \in \mathscr{G} \mid g_{1}(z, w) \in D_{\varrho}\left(z_{0}=r^{\prime} \in \mathbb{C}\right) \text { and }\left\|\left(g_{2}, \ldots, g_{n}\right)(z, w)\right\|<\varepsilon_{3} r\right. \\
& \left.\quad \text { for all }(z, w) \in \mathbb{C} \times \mathbb{C}^{n}\right\}
\end{aligned}
$$

where $\varrho=\frac{r^{\prime}-\bar{r}}{2}$ and $D_{\varrho}\left(z_{0}=r^{\prime}\right)$ is the disk of radius $\varrho$ of $\mathbb{C}$ centered at the real point $z_{0}=r^{\prime}$. The equation $\bar{\partial}_{J} f=g$ on $U \times \mathscr{N}_{0} \times V$ gives (compare Polterovich [9], App.):

$$
\begin{aligned}
\pi\left(r^{\prime}-\varrho\right) & \leq\left|\int_{D^{2}} g_{1}(z, f(z))\right| \leq\left|\frac{1}{2} \int_{D^{2}}\left(\frac{\partial f_{1}}{\partial x}+i \frac{\partial f_{1}}{\partial y}\right)\right|+\left|\int_{D^{2}}\left(g_{1}(z, f(z))-\bar{\partial}_{J_{0}} f_{1}\right)\right| \\
& \leq \pi \max _{z \in \partial D^{2}}\left|f_{1}(z)\right|+\varepsilon_{1} \int_{D^{2}}\|D f\|
\end{aligned}
$$

and

$$
\begin{aligned}
\int_{D^{2}}\|D f\| & \leq C_{1} \int_{D^{2}}\|D f\|_{J} \leq C_{1} \sqrt{\pi}\left(\int_{D^{2}}\|D f\|_{J}^{2}\right)^{1 / 2} \\
& \leq C_{1} \sqrt{\pi}\left(\int_{D^{2}}\|g(z, f(z))\|_{J}^{2}\right)^{1 / 2} \\
& \leq C_{2} \sqrt{\pi}\left(\int_{D^{2}}\|g\|^{2}\right)^{1 / 2} \leq \pi C_{3} r
\end{aligned}
$$


by Hölder's inequality and (2), where

$$
C_{3} \rightarrow 1 \text { when } \varepsilon_{1}, \varepsilon_{2}, \varepsilon_{3} \rightarrow 0 \text {. Thus } \pi\left(r^{\prime}-\varrho\right) \leq \pi \bar{r}+\pi \varepsilon_{1} C_{3} r
$$

which boils down to $\varrho \leq \varepsilon_{1} C_{3} r$ since $r^{\prime}-\varrho=\bar{r}+\varrho$. Consequently, there is no solution if $\varepsilon_{1}, \varepsilon_{2}, \varepsilon_{3}$ are sufficiently small.

Let us now fix a generic value $J \in U$ (regular for all $\pi_{\alpha}$ ) and a generic path $\gamma$ in $\mathscr{G}$ joining 0 to a generic $g \in V$ [regular value of $\bar{\partial}_{J}: \mathscr{N}_{\beta} \rightarrow \mathscr{G}$ for all $\beta \in$ $\left.H_{1}\left(L_{r} ; \mathbb{Z}\right)\right]$ such that $\|g(z, w)\|_{J} \leq r^{\prime}$ for all $(z, w) \in \mathbb{C} \times \mathbb{C}^{n}$.

Consider now the map $\bar{\partial}_{J}: \mathscr{N}_{0} \rightarrow \mathscr{G}$ and its restriction to $Y_{0, \gamma}=\bar{\partial}_{J}^{-1}(\gamma)$. On the one hand, the inverse image $\bar{\partial}_{J}^{-1}(0)$ is diffeomorphic to $L_{r}$. Indeed, for any map $f: D^{2} \rightarrow \mathbb{C}^{n}$,

$$
\begin{aligned}
\mid \frac{\partial f}{\partial x_{1}} & \left.\wedge \frac{\partial f}{\partial x_{2}}\right|_{J} ^{2}-\left(\omega\left(\frac{\partial f}{\partial x_{1}}, \frac{\partial f}{\partial x_{2}}\right)\right)^{2} \\
= & \operatorname{det}\left(\omega\left(\frac{\partial f}{\partial x_{i}}, J \frac{\partial f}{\partial x_{j}}\right)\right)_{1 \leq i, j \leq 2}-\left(\omega\left(\frac{\partial f}{\partial x_{1}}, \frac{\partial f}{\partial x_{2}}\right)\right)^{2} \\
= & \left\|\frac{\partial f}{\partial x_{1}}\right\|_{J}^{2}\left\|\frac{\partial f}{\partial x_{2}}\right\|_{J}^{2}-\left\langle\frac{\partial f}{\partial x_{1}}, \frac{\partial f}{\partial x_{2}}\right\rangle_{J}^{2}-\left\langle J \frac{\partial f}{\partial x_{1}}, \frac{\partial f}{\partial x_{2}}\right\rangle_{J}^{2} \geq 0 . \\
& \left(x_{1}=x, x_{2}=y\right)
\end{aligned}
$$

(One easily checks this inequality for $J=J_{0}$, and afterwards for any compatible $J$ using a linear isometry which maps $\mathbb{R}\left\langle\partial f / \partial x_{1}, J \partial f / \partial x_{1}, \partial f / \partial x_{2}\right\rangle$ onto $\left(\mathbb{C}, J_{0}\right) \times \mathbb{R}$ and is pseudoholomorphic on $\mathbb{R}\left\langle\partial f / \partial x_{1}, J \partial f / \partial x_{1}\right\rangle$.) Thus

$$
|\partial f / \partial x \wedge \partial f / \partial y|_{J} \geq|\omega(\partial f / \partial x, \partial f / \partial y)|
$$

with equality if $f$ is $J$-holomorphic. Denote by $a_{J}(f)$ and $a_{s}(f)=\int f^{*}(\omega)$ the area of $f$ with respect to the norm \|\|$_{J}$ and the symplectic area of $f$. Hence

$$
a_{J}(f) \geq\left|a_{s}(f)\right|
$$

and

$$
a_{J}(f)=a_{s}(f) \text { if } f \text { is } J \text {-holomorphic . }
$$

Since any contractible disk $f:\left(D^{2}, \partial D^{2}\right) \rightarrow\left(\mathbb{C}^{n}, L_{r}\right)$ satisfies $0=\int_{\partial D^{2}} f^{*} \lambda=$ $\int f^{*} \omega$, such a disk cannot be $J$-holomorphic unless it is constant. Thus $\bar{\partial}_{J}^{-1}(0) \simeq L_{r}$. $D^{2}$

On the other hand, $Y_{0, \gamma}$ cannot be compact because compactness would imply, by the Sard-Smale theorem [13], that $Y_{0, \gamma}$ is a cobordism between $\bar{\partial}_{J}^{-1}(0) \simeq L_{r}$ and $\bar{\partial}_{J}^{-1}(g)=\emptyset$, and the image of $Y_{0, \gamma}$ by the evaluation map at $z=1 \in \partial D^{2}$, $e v_{1}: Y_{0, \gamma} \rightarrow L_{r}$, would then show that $\left[\operatorname{ev}_{1}\left(\bar{\partial}_{J}^{-1}(0)\right)\right]=\left[L_{r}\right]$ is zero when considered as an element of $H_{n}\left(L_{r} ; \mathbb{Z} / 2 \mathbb{Z}\right)$. Therefore $Y_{0 . \gamma}$ is not compact and there exists a $C^{\infty}$ divergent sequence $\left\{f_{i}\right\} \subset Y_{0, \gamma}$ (containing no convergent subsequence). As in 
Sikorav [11], the compactness of $\gamma$ and $L_{r}$, and the fact that $L_{r}$ is Lagrangian imply that $\left\{f_{\imath}\right\}$ is $C^{0}$-bounded and that their $J$-areas are bounded since:

$$
\begin{aligned}
\int_{D^{2}}\left\|\frac{\partial f}{\partial x} \wedge \frac{\partial f}{\partial y}\right\|_{J} & \leq \int_{D^{2}} \frac{1}{2}\left(\left\|\frac{\partial f}{\partial x}\right\|_{J}^{2}+\left\|\frac{\partial f}{\partial y}\right\|_{J}^{2}\right) \\
& =\int_{D^{2}} 2\|D f(z)\|_{J}^{2}=\int_{D^{2}} 2\left\|\bar{\partial}_{J} f\right\|_{J}^{2} \quad \text { according to (2) },
\end{aligned}
$$

hence

$$
a_{J}\left(f_{i}\right) \leq 2 \pi r^{\prime 2} \text { for all } i
$$

We can therefore apply Gromov's compactness theorem (see [6], $1.5 D_{1}, 1.5 D_{2}$, and $1.5 E_{1}$ ): there exists a subsequence of $\left\{f_{\imath}\right\}$, that we still denote by $\left\{f_{i}\right\}$, converging to a cusp curve

$$
f_{\infty}=f+\sum_{\jmath=1}^{k} h_{\jmath} \quad(k \geq 1),
$$

where the $h_{\jmath}$ are non-constant (maybe multiply covered) $J$-holomorphic disks with boundaries in $L_{r}$ appearing by concentration of curvature in the neighbourhood of $l$ points $\left\{z_{1}, \ldots, z_{l}\right\} \subset \partial D^{2}(l \leq k$ since there might be several disks bubbling off at the same point), and where $\bar{f}$ is a disk whose boundary lies in $L_{r}$ and such that $\bar{\partial}_{J} f \in \gamma$. The precise configuration of attaching points among the disks $f, h_{1}, \ldots, h_{k}$, and the reparametrizations needed to give a correct account of the weak convergence $f_{\imath} \rightarrow f_{\infty}$ may be found in the detailed study [15]. We will simply need here the fact that there is no loss of energy during the bubbling off phenomenon. Thus:

Proposition 1 (Sikorav [11]). The sum of the symplectic areas of the $h_{j}$ is smaller than $\pi r^{2}$.

This is a consequence of:

$$
2 \sum_{j=1}^{k} a_{s}\left(h_{j}\right) \leq \lim _{i} a_{J}\left(f_{i}\right)<2 \pi r^{2} .
$$

The last inequality follows from (4). For the first one, let $\left(U_{i j^{\prime}}\right)_{i \in \mathbb{N}, 1 \leq j^{\prime} \leq \ell}$ be a sequence of neighbourhoods of $z_{j^{\prime}} \in \partial D^{2}$ such that, up to reparametrization,

$$
\lim _{i \rightarrow \infty} a_{J}\left(\left.f_{\imath}\right|_{j^{\prime}} U_{\imath j^{\prime}}\right)=\sum_{j=1}^{k} a_{J}\left(h_{j}\right) .
$$

Then the same equality holds for the symplectic area. On the other hand,

$$
\begin{aligned}
a_{J}\left(f_{i}\right) & =a_{J}\left(\left.f_{i}\right|_{J^{\prime}} U_{i j^{\prime}}\right)+a_{J}\left(\left.f_{i}\right|_{D^{2} \backslash \bigcup_{J^{\prime}} U_{\imath j^{\prime}}}\right) \\
& \geq a_{s}\left(\left.f_{i}\right|_{j^{\prime}} U_{i j^{\prime}}\right)+a_{s}\left(\left.f_{i}\right|_{D^{2} \backslash \bigcup_{j^{\prime}} U_{\imath J^{\prime}}}\right) \geq 2 a_{s}\left(\left.f_{i}\right|_{j^{\prime}} U_{\imath \jmath^{\prime}}\right)
\end{aligned}
$$

by (3) and the fact that $\int_{D^{2}} f_{i}^{*}(\omega)=0$. Taking the limit gives the required inequality. 
Let us now find bounds for the Maslov indexes of the boundaries of the $h_{j}$. Since $f_{\infty}=f+\sum_{j=1}^{k} h_{j}$ comes from a sequence of contractible disks and since non-constant $J$-holomorphic disks have positive symplectic areas, then $h_{j} \in M_{\alpha_{j}}$ and $f \in F_{\beta}$, where $\beta, \alpha_{1}, \ldots, \alpha_{k} \neq 0$ and

$$
\mu(\beta)+\sum_{\jmath=1}^{k} \mu\left(\alpha_{j}\right)=0
$$

Finally, the choices of $J$ and $\gamma$ imply $\mu(\beta) \geq-n-1$ and $\mu\left(\alpha_{\jmath}\right) \geq-n+3$. This gives the following:

Proposition 2 (compare Polterovich [9]). There exists a J-holomorphic disk whose boundary is a loop $\alpha$ in $L_{r}$, such that:

$$
-n+3 \leq \mu(\alpha) \leq n+1
$$

These two propositions lead to the following:

Theorem 2. Let $L_{r}$ be a closed Lagrangian submanifold in $D(r) \times \mathbb{C}^{n-1}$. Then there exists a class $\alpha \in H_{1}\left(L_{r} ; \mathbb{Z}\right)$ with

$$
0<\lambda(\alpha)<\pi r^{2} \text { and }|\mu(\alpha)| \leq n+1
$$

Now suppose that $L$ is any closed Lagrangian submanifold in $\mathbb{C}^{n}$ such that $\left.\lambda(L)\right|_{\operatorname{ker} \mu(L)}$ is rational. There exists a basis $\left\{\beta_{1}, \ldots, \beta_{m}\right\}$ of $H^{1}(L ; \mathbb{Z})$ in which $\lambda(L)$ and $\mu(L)$ can be expressed as:

$$
\begin{aligned}
& \lambda(L)=\delta\left(x \beta_{1}+\sum_{i=2}^{m} p_{i} \beta_{i}\right), \\
& \mu(L)=l \beta_{1},
\end{aligned}
$$

where $\delta \in \mathbb{R}_{+}=(0, \infty), x \in \mathbb{R}, l, p_{\imath} \in \mathbb{N}$, and $\sum_{i=2}^{m} p_{\imath} \beta_{\imath}$ is a primitive class of the zero class of $H^{1}(L ; \mathbb{Z})$. Suppose first that $\mu(L) \neq 0$ and let $q \in \mathbb{N}$ be the largest integer such that $q l \leq n+1$. Set

$$
\varepsilon(x, q)=\inf \left[\left\{\left|a_{1} x+\sum_{i=2}^{m} a_{i} p_{i}\right| \text { such that } a_{1}, \ldots, a_{m} \in \mathbb{Z},\left|a_{1}\right| \leq q\right\} \backslash\{0\}\right]>0
$$

Since $\operatorname{gcd}\left(p_{2}, \ldots, p_{m}\right)=1, \varepsilon(x, q)$ is the smallest non-zero difference between one of the multiples $\{0, x, \ldots, q x\}$ and an integer. By construction, $\delta \varepsilon(x, q)$ is the smallest positive symplectic area allowed by the constraint on the Maslov index of Theorem 2. 
Therefore, there is no Hamiltonian isotopy mapping $L$ into a tube $D(r) \times \mathbb{C}^{n-1}$ whenever $r \leq(\delta \varepsilon(x, q) / \pi)^{1 / 2}$, that is:

$$
R(L) \geq(\delta \varepsilon(x, q) / \pi)^{1 / 2} .
$$

Finally, if $\mu(L)=0, R(L) \geq(\varrho / \pi)^{1 / 2}$, where $\varrho$ is the largest real number such that $\lambda(L)=\varrho \times$ (integral form). Q.E.D.

Remark. When $\mu(L) \neq 0$, the lower bound $(\delta \varepsilon(x, q) / \pi)^{1 / 2}$ is in general better than $(\varrho / \pi)^{1 / 2}$ even in the rational case $(x \in \mathbb{Q})$. Here is an example: let $p$ be a positive integer, $N \geq 3$ an integer, $S^{1}\left((1 / \pi)^{1 / 2}\right)$ and $S^{1}\left(\left(\frac{p(N+1)-1}{p \pi}\right)^{1 / 2}\right)$ the circles in $\mathbb{C}$ of areas 1 and $\frac{p(N+1)-1}{p}$, and $T^{2} \hookrightarrow \mathbb{C}^{2}$ their product. In the basis $\left\{e_{1}^{*}, e_{2}^{*}-e_{1}^{*}\right\}$ of $H^{1}\left(T^{2} ; \mathbb{Z}\right)$,

$$
\lambda\left(T^{2}\right)=\frac{p N-1}{p}\left(\frac{a}{p N-1}, 1\right) \text { and } \mu\left(T^{2}\right)=(2,0) .
$$

The smallest positive value of $\lambda\left(T^{2}\right)$, that is $1 / p$, is realized only on classes with too large Maslov indexes. In fact, the smallest positive symplectic area among classes $\alpha$ of Maslov index $\mid(\mu(\alpha) \mid \leq 3$, is

$$
\delta \varepsilon\left(x=\frac{p}{p N-1}, q=1\right)=\left(\frac{p N-1}{p}\right)\left(\frac{p}{p N-1}\right)=1 .
$$

So that the lower bound $(\varrho / \pi)^{1 / 2}$ gives $R\left(T^{2}\right) \geq \sqrt{\frac{1}{p \pi}}$, whereas the lower bound $(\delta \varepsilon(x, q) / \pi)^{1 / 2}$ gives $\sqrt{\frac{1}{\pi}}$ which, in this case, is optimal: $R\left(T^{2}\right)=\sqrt{\frac{1}{\pi}}$.

Acknowledgements. I am grateful to the referee for pointing out several inaccuracies in the first version of this paper, and to A. Jaffe for his suggestion to publish this paper in CMP even though we agree that the subject of this article is at the borderline of those usually covered by this journal.

\section{References}

1. Arnold, V.I.: On a characteristic class entering in quantization conditions. Funct. Anal. Appl. 1, 1-13 (1967)

2. Ekeland, I.: Convexity methods in Hamiltonian mechanics. Ergebnisse der Mathematik vol. 19. Berlin, Heidelberg, New York: Springer 1990

3. Ekeland, I., Hofer, H.: Symplectic topology and Hamiltonian dynamics. I, II. Math. Z. 200, 355-378 (1989); Math. Z. 203, 553-568 (1990)

4. Floer, A.: The unregularized gradient flow for the symplectic action. Commun. Pure Appl. Math. 41 (1988)

5. Floer, A., Hofer, H.: In preparation

6. Gromov, M.: Pseudoholomorphic curves in symplectic manifolds. Invent. Math. 82, 307-347 (1985)

7. Hofer, H.: On the topological properties of symplectic maps. Preprint Essen-Bochum-Düsseldorf 1990 
8. McDuff, D.: Examples of symplectic structures. Invent. Math. 89, 13-36 (1987)

9. Polterovich, L.: The Maslov class of the Lagrange surfaces and Gromov's pseudoholomorphic curves. Trans. Am. Math. Soc. 325, 241-248 (1991)

10. Polterovich, L.: Symplectic displacement energy for Lagrangian submanifolds. Preprint IHES, 1991

11. Sikorav, J.C: Quelques propriétés des plongements lagrangiens. Preprint Université Paris-Sud, Orsay, 1990

12. Sikorav, J.C.: Systèmes hamiltoniens et topologie symplectique. Publication Dipartimento di Matematica, Universita di Pisa, ETS Editor, 1990

13. Smale, S.: An infinite dimensional version of Sard's theorem. Am. J. Math. 87, 861-866 (1965)

14. Viterbo, C.: Plongements lagrangiens et capacités symplectiques de tores dans $\mathbb{R}^{2 n}$. C.R. Acad. Sci. Paris, t.113, Série I, 487-490 (1990)

15. Wolfson, J.: Gromov's compactness of pseudo-holomorphic curves and symplectic geometry. J. Diff. Geom. 28, 383-405 (1988)

Communicated by A. Jaffe 